\title{
QUICK TURNAROUND POSITIVE POLARITY OPTIONS FOR MERCURY*
}

\author{
R.J. Allen ${ }^{\xi}$, R.J. Commisso, G.Cooperstein ${ }^{\text {a }}$, D.D. Hinshelwood, \\ S.L. Jackson, P.F. Ottinger ${ }^{\mathrm{a}}$, J.W. Schumer, \\ S.B. Swanekamp and J.C. Zier \\ Pulsed Power Physics Branch, Plasma Physics Division, \\ Naval Research Laboratory, Washington, DC 20375 USA
}

\section{Abstract}

Mercury, a 2-TW inductive voltage adder located at the Naval Research Laboratory in Washington, DC, had previously been converted from negative to positive polarity output by rotating each of the cells[1]. Positive polarity was needed to field an ion-beam diode.[2] However, rotating the cells takes about 2 to 3 weeks and is very labor intensive. So, when we next needed to operate in positive polarity, we considered two options to quickly turnaround Mercury to positive polarity; reverse charging the Marx and fielding a vacuum convolute.

Charging the Marx with the opposite polarity is the simplest way to operate Mercury in positive polarity. However, because the breakdown and flashover strengths of the components are lowered when the polarity is reversed in this way, it is required to limit the Marx charge to $2 / 3$ of its normal charging voltage to prevent failures. This limits the maximum output voltage, with the existing center conductor, to about 4.5 MV. An alternative approach we considered was to operate in negative polarity but to field a vacuum convolute inside our large diameter load chamber. Simulations and field calculations showed that this approach could work and the maximum output would be about $6 \mathrm{MV}$.

We chose to operate Mercury in positive polarity by reverse charging the Marx and successfully operated an ion diode at a lower power level. To improve the pulse shape, we modified the self-breaking PFL water switch hardware. After this and a few other changes, Mercury can now be switched to a reliable and repeatable positive polarity mode in days instead of weeks, although at a lower than maximum power level. Details and results will be presented.

\section{I.INTRODUCTION}

We have explored two options for operating the Mercury IVA (Inductive Voltage Adder)[1] in positive polarity that are quicker than our normal procedure of rotating all of the cells by 180 degrees. Rotating the cells can take 3 weeks to complete and another 3 weeks to

\footnotetext{
* Work supported by UK/AWE via DTRA

$\xi$ email: Raymond.allen@nrl.navy.mil

${ }^{a}$ Consultant to NRL through Engility, Chantilly, VA 20151
}

rotate back to negative polarity. This is a burden for short experimental runs in positive polarity when most of our experiments require negative polarity. The two options we have considered for quick turnaround to positive polarity are a Marx polarity reversal and a vacuum convolute.

\section{II.MARX POLARITY REVERSAL}

One fast way to change Mercury from negative to positive polarity is to simply reverse the positive and negative Marx capacitor power supply connections. The main disadvantage to this approach is that we should only charge the Marx to $2 / 3$ of the nominal $75 \mathrm{kV}$. This is because the various water gaps, oil gaps and insulators in the machine have a lower breakdown threshold with the center conductors at positive polarity. The machine configuration is then very similar to $50 \mathrm{kV}$ Marx charge shots that are occasionally taken in negative polarity for calibration of current monitors. The main difference is that we had to reduce the twelve self-breaking PFL (pulse-forming line) electrode gaps from $30 \mathrm{~mm}$ (usual spacing for $50-\mathrm{kV}$ operation) to $15 \mathrm{~mm}$. Spacers had to be added to allow the switches to achieve this small gap. Other setup differences are that the core reset voltage polarity is reversed and we apply graphite spray to the outer conductor of the MITL (magnetically-insulated transmission line) instead of the inner conductor. The Mercury PFLs also contain a self-breaking prepulse switch section, as shown in Fig.1, but we did not change this gap spacing from the usual $10 \mathrm{~mm}$ setting for $50 \mathrm{kV}$.

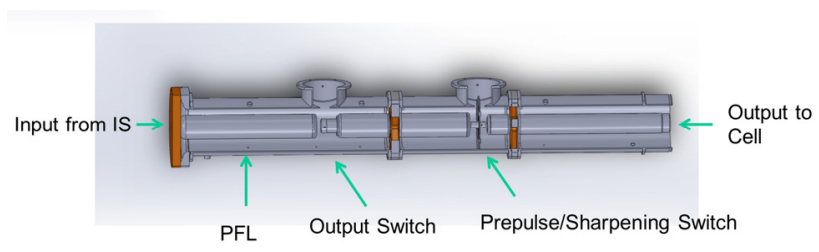

Figure 1. Cutaway view of a Mercury PFL with the main parts identified. 
Although the machine behaved reasonably well with the PFL gaps reduced to $15 \mathrm{~mm}$, the PFL switch jitter noticeably increased. At full power, this jitter could have led to vacuum insulator damage. To reduce jitter and thereby improve reproducibility in the output waveshape, we fabricated new PFL output switch electrode hardware that put the enhanced electrode on the opposite side of the switch. The upstream side of the PFL output switch, negative-voltage in negative polarity, is normally a flat plate, while the downstream side (positive wrt the upstream side) is a smaller diameter hollow cylinder with sharp edges at inner and outer radii. As shown in Fig.2c, the new, positive-polarity hardware allowed us to move the sharp, field enhanced, electrode to the upstream side of the switch (now positive). To save costs, the downstream side was not made to a flat plate, but just made less field enhanced.
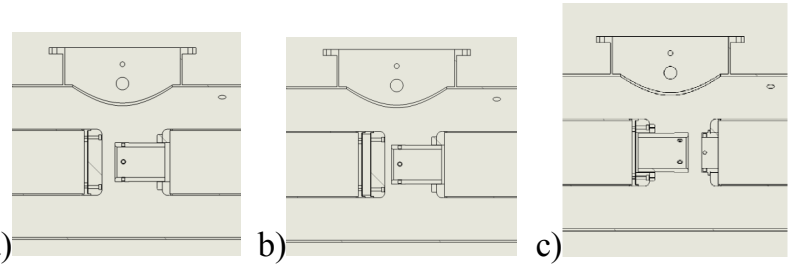

Figure 2. PFL output switch cross-sectional diagrams for a) the original switch configuration with $30-\mathrm{mm}$ electrode spacing, b) with a spacer added to give a 15 -mm electrode spacing, and c) with new hardware to place the field enhanced electrode up the upstream side of the switch (upstream to the left)

With the new hardware, the correct timing was again achieved with a $30-\mathrm{mm}$ electrode spacing. Also, the jitter was noticeably reduced to near the negative polarity level. Representative waveforms from the twelve PFL output voltage monitor signals (vols) are shown in Fig. 3. The standard deviations in the PFL timings for several shots, after accounting for deliberate staggering, $\sigma$, are shown in Fig. 4. We stagger the timings by making \pm 1 -mm adjustments to the nominal PFL electrode spacings in an attempt to have 2-ns delays between the six induction cells (each fed by two PFLs). Note that a 6-ns delay between the first six and last six PFLs is made by adjusting the IS (intermediate store) laser switch laser timings. So, the corrected timing jitter was calculated by adding 5 ns to vols $1 \& 2$ (feeding cell \#1), adding 3 ns to vols $3 \& 4,1$ ns to vols $5 \& 6,-1$ ns to vols $7 \& 8,-3$ ns to vols $9 \& 10$, and -5 ns to vols $11 \& 12$ and then taking the standard deviation of the times they each reach a threshold voltage during the initial rise of the pulse. This jitter measurement for a series of shots in negative polarity at $50 \mathrm{kV}$ (with an average value of $2.77 \mathrm{~ns}$ ), positive polarity with electrode spacer (average of 5.21 ns), and positive polarity with the new electrode hardware (average of $3.26 \mathrm{~ns}$ ) is shown in Fig. 4. Note that this jitter measurement also includes the jitter in the
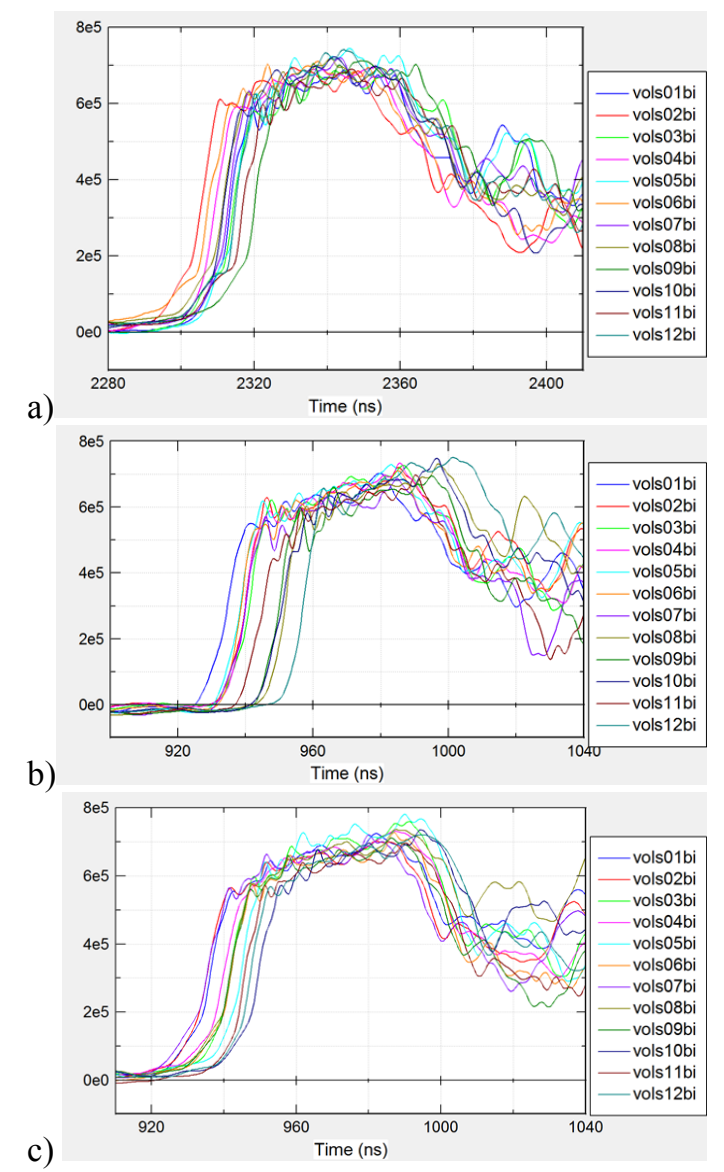

Figure 3. PFL output waveforms (vols) for representative shots in a) negative polarity (shot 0730), b) positive polarity with electrode spacers (shot 1086) and c) positive polarity with new electrode hardware (shot 1117).

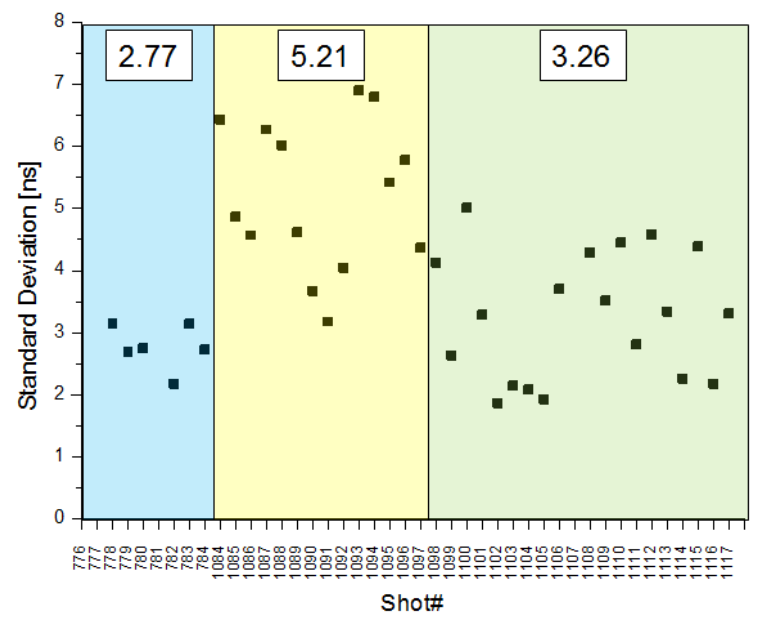

Figure 4. Calculated PFL output jitter, $\sigma$, for three series of $50 \mathrm{kV}$ shots in (left) negative polarity, (middle) positive polarity with spacers and (right) positive polarity with new electrode hardware. 
four laser triggered switches that connect IS bottles to the PFLs. An increase in this laser switch jitter in positive polarity could explain why the jitter with the new electrode hardware is slightly above that from negative polarity.

\section{III.VACUUM CONVOLUTE}

A vacuum convolute was explored as another way of quickly fielding a positive polarity diode. In this approach, the machine is operated in the usual negative polarity mode, but polarity is reversed in the load chamber at the output of the machine. The post-hole convolute works by placing rods (posts) at the end of the center conductor that go through holes in a ground plate and then connect to the cathode of the diode as shown in Fig. 5.

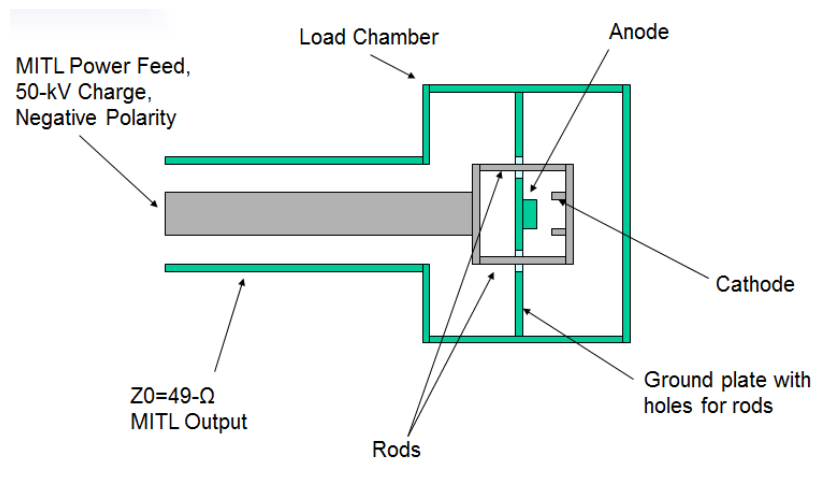

Figure 5. Diagram of a proposed vacuum post-hole convolute in the Mercury load chamber.

Convolutes are routinely used in low impedance machines where losses at the convolute are not critical.

However, in a relatively high impedance machine like Mercury, it is critical that there be low losses in the convolute. To minimize losses we would use toroidal

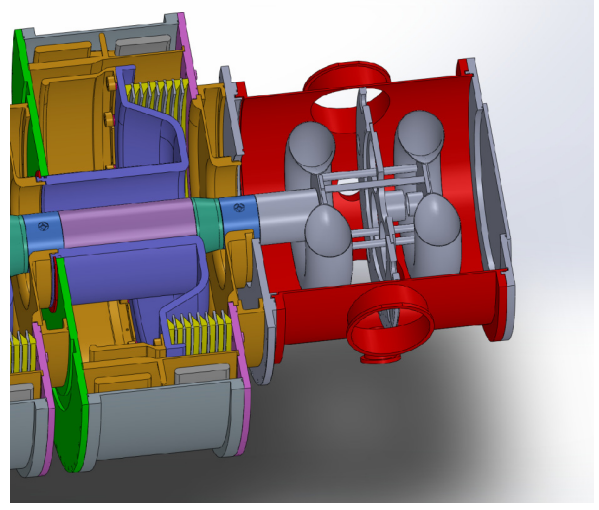

Figure 6. Cutaway view of possible convolute hardware with toroidal field shapers and large rods and holes. field shapers on either side of the rods as shown in Fig. 6. Also, we could use large diameter rods to perhaps prevent electron emission from the rods. Large holes in the ground plate could be used to ensure magnetic insulation.

Electrostatic field calculations in 2D show that with special preparation of the toroidal field shapers to prevent electron emission up to $800 \mathrm{kV} / \mathrm{cm}$, the convolute could work at up to $6 \mathrm{MV}$. A field plot of the geometry in Fig. 6 is shown in Fig. 7.
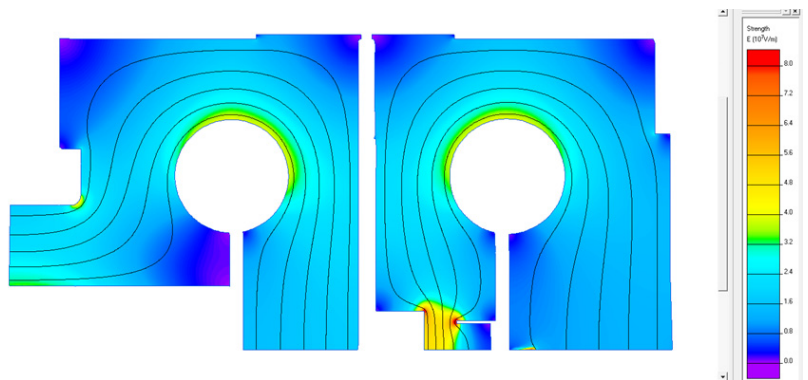

Figure 7. A 2 D electrostatic field plot showing the electric field in the load chamber.

The PIC (particle-in-cell) code LSP[3] was used to simulate this convolute in $3 \mathrm{D}$. One of the simulated geometries is shown in Fig. 8 where eight-fold symmetry was used to speed the computation. The input to the simulation was a $10-\mathrm{ns}$ ramp up to $4 \mathrm{MV}$ from a $20-\Omega$ source to approximate Mercury. With a simple, $12.5-\Omega$, electron only diode load there is very little loss in the convolute and the diode voltage is $2.5 \mathrm{MV}$ at $200 \mathrm{kA}$. There is very little loss even with the rods allowed to emit electrons. A particle plot of this situation is shown in Fig. 9 with center conductor electron particles in blue, rod electrons in green and diode electrons in red.

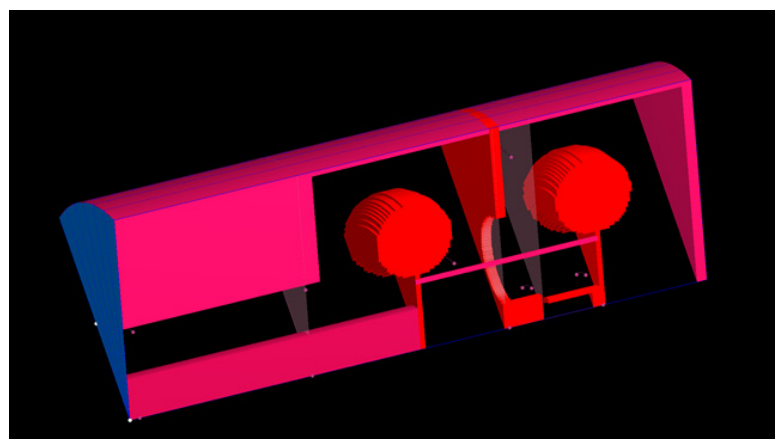

Figure 8. Geometry used in an LSP simulation of the vacuum convolute. 


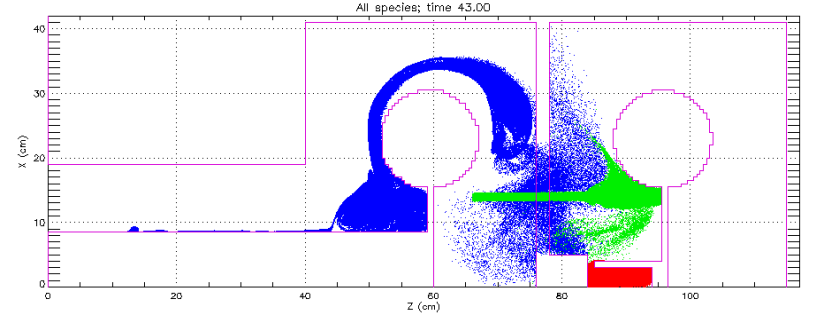

Figure 9. A particle plot from LSP showing center conductor electrons in blue, rod electrons in green and diode electrons in red.

\section{IV.SUMMARY}

We have found that we can more quickly change Mercury to positive polarity by simply reversing the Marx charging voltage. The main limitation with this approach is that we can only safely operate at a reduced Marx charging voltage. New water switch electrode hardware was required to reduce the water switch jitter to acceptable levels.

A second approach that was considered was to use a vacuum convolute in the load chamber to invert the polarity. Some limited simulations have shown that this approach can work well with a low impedance diode load.

\section{V.REFERENCES}

[1] R.J. Allen, et al., "Conversion of Mercury (a 2-TW inductive voltage adder) to positive polarity," 2009 IEEE Pulsed Power Conference, pp.1171-1175, June 28 2009July 22009

[2] D.D. Hinshelwood, et al., "Ion diode performance on a positive polarity inductive voltage adder with layered magnetically insulated transmission line flow", Phys.

Plasmas 18, 053106 (2011)

[3] LSP is a software product of ATK Mission Research, Albuquerque, NM 87110. 